FINAL REPORT

\title{
FAST COMPUTATIONAL ALGORITHMS FOR PARTIAL DIFFERENTIAL EQUATIONS AND UNCERTAINTY QUANTIFICATION
}

\author{
Howard C. Elman \\ Department of Computer Science and \\ Institute for Advanced Computer Studies \\ University of Maryland \\ College Park, MD 20742
}

January 2013

An overview of our accomplishments during the final term of this grant (1 September 2008 - 30 June 2012) is given below. These fall mainly into three categories: fast algorithms for linear eigenvalue problems; solution algorithms and modeling methods for PDEs with uncertain coefficients; and preconditioning methods and solvers for models of computational fluid dynamics (CFD). Publications referenced are listed immediately below.

\section{Main Accomplishments}

Fast eigenvalue solvers (Publications P1, P6, P9, P10, P12, P15, P17). Results include detailed analysis of the performance of Rayleigh quotient iteration for finding the smallest eigenvalues of positive-definite matrices when required matrix solves are replaced by approximate (inexact) computations (P1); development of robust preconditioning strategies for use in combination with subspace iteration (P6) and Arnoldi iteration (P9) for eigenvalue problems; and demonstration of the efficient use of new methods for computing eigenvalues associated with Hopf bifurcation in stability analysis of dynamical systems (P10, P12, P17).

Computational algorithms for PDEs with uncertain coefficients (P3, P5, P8, P11, P13, P16). Progress in this area includes several studies of the diffusion equation where the diffusion coefficient is a random field. In particular, we have developed an efficient preconditioner for the first-order (Darcy) formulation of the problem derived from a multigrid method designed to handle $\mathrm{H}$ (div) operators weighted by random diffusion coefficients; analysis and experimental results establish textbook multigrid convergence (P3). We have performed a careful study (P5) comparing the performance of the stochastic Galerkin and stochastic collocation methods for solving the stochastic diffusion equation, showing that if the dependence on stochastic parameters is linear, then the Galerkin method can be significantly more effective when a good (multigrid) preconditioner is used for the coupled Galerkin system. We have also developed an efficient method for handling such problems when the coefficient is of log-normal form (a particular example of a nonlinear function of the parameters); in this case, we take advantage of the fact that the diffusion problem can be transformed to an equivalent convection-diffusion problem depending linearly on the coefficients (P8). Finally, we have shown that the performance of collocation methods can be improved using adaptive strategies, and that methods of kernel density estimation can be used to estimate statistical quantities in cases when joint densities are not available (P11).

Preconditioners and solvers for models of CFD (P2, P4, P7). We have made a careful determination of the effects of boundary conditions on the performance of preconditioners for the 
incompressible Navier-Stokes equations, leading to significant improvements in performance (P2). In addition, we have shown that the preconditioning methods developed through this project are robust and easily adapted to sophisticated models, including one concerned with bouyancy driven flow that includes thermal effects in the model (P7), and another simulating microfluidic flows driven by an electrokinetic process (P4).

\section{Publications}

P1. F. Xue and H. C. Elman, Convergence analysis of iterative solvers in inexact Rayleigh quotient iteration, SIAM J. Matrix Anal. Appl. 31:877-899, 2009.

P2. H. C. Elman and R. S. Tuminaro, Boundary conditions in approximate commutator preconditioners for the Navier-Stokes equations, Electronic Transactions on Numerical Analysis 35:257280, 2009.

P3. H. C. Elman, D. G. Furnival and C. E. Powell, H(div) preconditioning for a mixed finite element formulation of the diffusion problem with random data, Mathematics of Computation 79:733-760, 2010.

P4. R. R. Shuttleworth, H. C. Elman, K. R. Long and J. A. Templeton, Fast solvers for models of ICEO microfluidic flows, International Journal for Numerical Methods in Fluids 65:383-404, 2011.

P5. H. C. Elman, C. W. Miller, E. T. Phipps and R. S. Tuminaro, Assessment of collocation and Galerkin approaches to linear diffusion equations with random data, International Journal for Uncertainty Quantification 1:19-34, 2011.

P6. F. Xue and H. C. Elman, Fast inexact subspace iteration for generalized eigenvalue problems with spectral transformation, Linear Algebra and Its Applications 435:601-622, 2011.

P7. H. Elman, M. Mihajlović and D. Silvester, Fast iterative solvers for buoyancy driven flow problems, Journal of Computional Physics 230:3900-3914, 2011.

P8. E. Ullmann, H. C. Elman and O. G. Ernst, Efficient iterative solvers for stochastic Galerkin discretizations of log-transformed random diffusion problems, SIAM Journal on Scientific Computing 34:A659-A682, 2012.

P9. F. Xue and H. C. Elman, Fast inexact implicitly restarted Arnoldi method for generalized eigenvalue problems with spectral transformation, SIAM Journal on Matrix Analysis and Applications 33:433-459, 2012.

P10. H. C. Elman, K. Meerbergen, A. Spence and M. Wu, Lyapunov inverse iteration for identifying Hopf bifurcations in models of incompressible flow, SIAM Journal on Scientific Computing 34:A1585-A1606, 2012.

P11. H. C. Elman and C. W. Miller, Stochastic collocation with kernel density estimation, Computer Methods in Applied Mechanics and Engineering 245-245:36-46, 2012.

P12. H. C. Elman and M. Wu, Lyapunov inverse iteration for computing a few rightmost eigenvalues of large generalized eigenvalue problems UMIACS-TR-2012-07, 2012.

P13. H. C. Elman and Q. Liao, Reduced basis collocation methods for partial differential equations with random coefficients UMIACS-TR-2012-09, 2012,

P14. C. E. Powell and E. Ullmann, Preconditioning stochastic Galerkin saddle point systems, SIAM Journal on Matrix Analysis and Applications 31:2813-2840, 2010.

P15. F. Xue, Numerical Solution of Eigenvalue Problems with Spectral Transformations, Doctoral Dissertation, 2009.

P16. C. Miller, Fast Algorithms for the Solution of Stochastic Partial Differential Equations, Doctoral Dissertation, 2012. 
P17. M. Wu, Linear Stability Analysis using Lyapunov Inverse Iteration, Doctoral Dissertation, 2012.

\section{Presentations of Results}

Int'l Conference on Approximation in Scientific Computing, Beijing, China, Oct. 2008.

Int'l Conference on Numerical Algebra and Scientific Computing, Nanjing, China, Nov. 2008. 34th Woudschoten Conference on Scientific Computing, Zeist, The Netherlands, Oct. 2009.

Workshop on Uncertainty Quantification, Royal Society of Edinburgh, Scotland, May 2010.

Workshop on Linear Algebra Aspects of Solving PDEs with Random Data, Manchester, England, Jan. 2012.

SIAM Annual Meeting, Denver, CO, July 2009.

SIAM Conference on Parallel Processing, Seattle, WA, Feb. 2010.

ICIAM 2011, Vancouver, CA (two minisymposium presentations), July 2011.

Copper Mountain Conference on Iterative Methods, Copper Mountain, CO, April 2012.

Conference in honor of Tony Chan's 60th birthday, UCLA, Los Angeles, CA, June 2012.

New York University (Courant Institute), Numerical Analysis Seminar, Jan. 2009, March 2012.

Purdue University, Mechanical Engineering Seminar, Feb. 2010.

University of Delaware, Analysis and PDE Seminar, April 1010.

NIST, Mathematical and Computational Sciences Division Seminar, June 1010.

Temple University, Applied Mathematics and Scientific Computing Seminar, March 2011.

Computational Science and Engineering Symposium, University of Illinois, April 2011.

Virginia Polytechnic Institute, Mathematics Colloquium, Nov. 2011.

Worcester Polytechnic Institute, Mathematics Colloquium, Dec. 2011.

University of Freiberg, Germany, Numerical Analysis Seminar, Jan. 2012.

Numerical Analysis Seminar, remote presentation to consortium of UK universities (Oxford, Bath, Bristol, Imperial and Warwick), May 2012.

\section{Personnel}

The grant supported Howard Elman (PI) and four graduate students in Maryland's Applied Mathematics and Scientific Computing Program: Fei Xue (Ph. D. 2009), Christopher Miller (Ph. D. 2012), Minghao Wu (Ph. D. 2012), and Edward Phillips. It also supported two postdoctoral appointments, Elisabeth Ullmann (April - September, 2009) and Qifeng Liao (February 2010 - June 2012). In addition, it supported two visits from visiting professor Alastair Spence from the University of Bath, England, in January 2009 and June 2010. 